\title{
Cinética de la Combustión Sin Llama de Gas Natural sobre CaOMgO
}

Elías de J. Gómez, Mauricio E. Sánchez y Javier A. Jaramillo

Universidad de Antioquia, Departamento de Ingeniería Química, Grupo Ciencia y Tecnología del Gas y Uso Racional de la Energía, Calle 67 № 53-108, bloque 18, oficina 435,Medellín-Colombia (e-mail: egomez@udea.edu.co,msanchez@udea.edu.co, ajarami@udea.edu.co)

\section{Resumen}

Se investigó la combustión sin llama de gas natural con aire en lecho fijo de $\mathrm{CaO} / \mathrm{MgO}$ en un reactor diferencial sin limitaciones difusionales, con el fin de obtener los parámetros cinéticos. El orden de reacción respecto al metano se determinó para concentraciones entre 1.5 y $3.0 \% \mathrm{v} / \mathrm{v}$ en mezclas aire-gas natural con oxígeno $100 \%$ exceso. Para hallar el orden de reacción respecto al oxígeno, se varió su concentración entre 6.0 y $9.0 \% \mathrm{v} / \mathrm{v}$ con $3.0 \% \mathrm{v} / \mathrm{v}$ de metano. La energía de activación y el factor pre-exponencial se determinaron con temperaturas entre 612.5 y $650^{\circ} \mathrm{C}$. Se obtuvo cinética tipo Langmuir-Hinshelwood-Hougen-Watson, órdenes de reacción uno respecto al metano y cero para el oxígeno, energía de activación $136.64 \mathrm{~kJ} / \mathrm{mol}$ y factor pre-exponencial global $15946 \mathrm{~mol} / \mathrm{g}$ min. Los resultados son útiles para diseñar sistemas de combustión de gas natural y depuradores de emisiones gaseosas que contengan metano.

Palabras clave: combustión sin llama, mezcla $\mathrm{CaO} / \mathrm{MgO}$, cinética, gas natural, reactor diferencial

\section{Kinetics of Natural Gas Flameless Combustion over $\mathrm{CaO} / \mathrm{MgO}$}

\begin{abstract}
Flameless natural gas combustion with air in a $\mathrm{CaO} / \mathrm{MgO}$ packed bed, operated as a differential reactor without diffusional limitations, was studied, in order to get kinetic parameters. Methane reaction order with concentrations between 1.5 and $3.0 \% \mathrm{v} / \mathrm{v}$ was determined using air-natural gas mixtures with $100 \%$ excess oxygen. In order to get the reaction order for oxygen, its concentration was varied between 6.0 and $9.0 \% \mathrm{v} / \mathrm{v}$ while methane was $3.0 \% \mathrm{v} / \mathrm{v}$. Activation energy and preexponential factor were determined for the temperature range 612.5 to $650^{\circ} \mathrm{C}$. LangmuirHinshelwood-Hougen-Watson type kinetics was obtained, reaction orders were one for methane and zero for oxygen, activation energy $136.64 \mathrm{~kJ} / \mathrm{mol}$ and pre-exponential factor $15946 \mathrm{~mol} / \mathrm{g} \mathrm{min}$. Results are useful in equipment design for natural gas combustion and for treating gaseous emissions containing methane.
\end{abstract}

Key words: flameless combustion, CaO/MgO mixture, kinetics, natural gas, differential reactor 


\section{INTRODUCCIÓN}

La combustión térmica de hidrocarburos tiene impactos negativos sobre el ambiente y la salud humana debido a las emisiones de inquemados, óxidos de nitrógeno y monóxido de carbono. Entre las alternativas para solucionar estos inconvenientes está el uso de superficies sólidas químicamente activas para la oxidación total de hidrocarburos, en mezclas de composición por debajo del límite inferior de inflamabilidad y a menor temperatura que la requerida en combustión térmica.

Los materiales más activos para combustión sin llama de gas natural son metales nobles y óxidos de metales de transición (Requies et al., 2008; Yoshida et. al., 2007; Gluhoi y Nieuwenhuys, 2007; Persson et al., 2007; Ramadj et al., 2007; Zhimin et al., 2007; Choudhary et al., 2002; Zwinkels et al., 1993, Pfefferle y Pfefferle, 1987). Los metales nobles son costosos, inestables a altas temperaturas y de poca disponibilidad. Los óxidos simples y mixtos de metales de transición, aunque de menor costo que los metales nobles, también presentan inestabilidad térmica, menor actividad y difícil preparación (Petrovic et al., 2008; Chiarello et al., 2006; Campagnoli et al., 2005; Chen et al., 2005). La necesidad de materiales activos, térmicamente estables y de menor costo, ha llevado al estudio de hexaaluminatos y óxidos de metales alcalinotérreos, los cuales son menos activos que los metales nobles, pero de mayor estabilidad térmica (Baylet et al., 2008; Li y Wang, 2007; Ren et al, 2007; Teng et al., 2007; Ersson et al., 2006; Choudhary et al., 2002; Berg y Järas, 1995). Los hexaaluminatos son un poco más activos pero de menor estabilidad térmica y más difícil preparación que el MgO, el más promisorio de los óxidos de metales alcalinotérreos (Berg y Järas, 1995).

La oxidación de metano sobre MgO puro o adicinado con otros elementos como $\mathrm{Li}$, Fe, Co y $\mathrm{Ca}$, viene aumentando en interés (Teng et al., 2007; Spretz et al., 2000; Berg y Järås, 1994; Aigler y Lunsford, 1991). El sistema Li/MgO muestra mayor selectividad hacia etano y etileno por acoplamiento oxidativo que a dióxido de carbono por oxidación total (Dubois y Cameron, 1990; Kimble y Kolts, 1987; Ito et al., 1985). Cuando al MgO se adiciona Fe o Co se da la oxidación total del metano promovida por el metal de transición (Ulla et al., 2001; Spretz et al., 2000). Algunos estudios plantean que la presencia de calcio en la superficie del MgO aumenta de manera considerable la actividad para oxidación parcial de metano (Aigler y Lunsford, 1991; Cunnigham et al., 1988; McCune y Wynblatt, 1983). Otros investigadores concluyeron que el MgO puro, usado frecuentemente como soporte de metales nobles por su alta estabilidad térmica, es activo para la oxidación total de metano en exceso de oxígeno (Teng et al., 2007; Berg y Järas, 1994). Gómez et al. $(2008,2007)$ obtuvieron alta actividad en combustión de metano y gas natural con aire sobre $\mathrm{CaO} / \mathrm{MgO}$ preparado a partir de $\mathrm{MgO}$ grado industrial con $\mathrm{CaO}$ como impureza.

En la literatura se reportan estudios cinéticos de la oxidación parcial de metano sobre MgO, Li/MgO y Fe/MgO (Spretz et al., 2000; Berg y Järas, 1994; Aigler y Lunnsford, 1991; Ito et al., 1985), pero no se hallan parámetros cinéticos para combustión de gas natural con exceso de oxígeno, sobre $\mathrm{CaO} / \mathrm{MgO}$.

En el presente trabajo se investigó la cinética de la combustión sin llama de mezclas gas natural-aire con concentraciones de metano por debajo del límite inferior de inflamabilidad, en lecho fijo empacado con partículas de material activo preparado por secado y calcinación del hidróxido; el cual se obtuvo a partir de la suspensión acuosa de $\mathrm{MgO}$ y $\mathrm{CaO}$. Se estudió la dependencia de la velocidad de reacción con respecto a la temperatura y a las concentraciones de metano y oxígeno bajo condiciones de reactor diferencial sin efectos difusionales. Los resultados obtenidos son fundamentales en el diseño de equipos para combustión sin llama de gas natural y de sistemas depuradores de emisiones gaseosas que contengan metano, sobre un material activo térmicamente estable, de bajo costo y fácil preparación.

\section{PARTE EXPERIMENTAL}

\section{Obtención de CaO/MgO y mezclas combustibles}

Se partió de óxido de magnesio grado industrial $\left(95.00 \% \mathrm{MgO} ; 0.90 \% \mathrm{CaO} ; 1.20 \% \mathrm{SiO}_{2} ; 0.50 \%\right.$ $\mathrm{Fe}_{2} \mathrm{O}_{3} ; 0.50 \% \mathrm{Al}_{2} \mathrm{O}_{3}$ y $1.84 \%$ inertes). El sólido activo se obtuvo por calcinación a $1000^{\circ} \mathrm{C}$ del hidróxido proveniente de la suspensión acuosa de la materia prima, mediante el procedimiento 
detallado por Gómez et al. (2007, 2006). Las mezclas combustibles de diversas composiciones se prepararon con gas natural de la Guajira, Colombia $\left(98.00 \% \mathrm{CH}_{4} ; 0.25 \% \mathrm{C}_{2} \mathrm{H}_{6} ; 0.05 \% \mathrm{C}_{3} \mathrm{H}_{8} ; 1.40 \%\right.$ $\mathrm{N}_{2} ; 0.14 \% \mathrm{CO}_{2}$ ), Oxígeno y Nitrógeno.

\section{Montaje experimental}

El esquema del montaje experimental se muestra en la Figura 1. Los flujos de mezcla reactiva se regularon con un controlador de flujo másico (OMEGA, modelo FMA-2606) y se calentaron con resistencia eléctrica a 220 VAC. Para mantener la temperatura de la mezcla gaseosa en la entrada al lecho se usó un controlador de temperatura PID (AUTONICS, modelo TZ4ST), con termopar tipo $\mathrm{K}$; como indicador de temperatura un termómetro digital (OMEGA, modelo HH2002AL). El sistema de reacción consistió de un tubo cerámico con longitud $220 \mathrm{~mm}$, diámetro interno $15 \mathrm{~mm}$ y espesor de pared $12 \mathrm{~mm}$, al cual se le instaló una resistencia eléctrica de $2 \mathrm{~kW}$ y cuatro termopares tipo $\mathrm{K}$ distribuidos uniformemente en su longitud. El reactor se recubrió con ladrillo refractario y manta cerámica. El tramo del tubo comprendido entre la entrada y el tercer termopar, usado para el control de temperatura, se llenó con cuarzo como material inerte, para facilitar la homogenización y el calentamiento de la mezcla reactiva; a partir de ahí, se empacaron las partículas de material activo, soportadas por un tapón cerámico perforado, ocupando una longitud de $38 \mathrm{~mm}$. Para dar estabilidad estructural al sólido y evitar que fuese arrastrado por el flujo, se le adecuó un tapón poroso de manta cerámica al final del lecho. La composición de los gases de combustión se midió con un analizador de gases infrarrojo no dispersivo, NDIR (MAIHAK, modelo Multor 610).

\section{Eliminación de limitaciones difusionales}

Para eliminar las diferencias de concentración entre el seno de la mezcla gaseosa reactiva y la superficie de las partículas (limitaciones externas), se mantuvo constante la relación entre la masa de material activo $(\mathrm{W})$ y el flujo molar de reactivos $\left(F_{0}\right)$, en $W / F_{o}=0.04 \mathrm{~g} \mathrm{~s} / \mathrm{mL}$. La concentración de metano en la entrada fue de $2.5 \% \mathrm{v} / \mathrm{v}$ y diámetro de partículas del lecho activo entre 1.6 y $2.0 \mathrm{~mm}$. Con el fin de determinar el flujo mínimo para el cual no se presentaran limitaciones de transporte externo, se midió la velocidad de consumo de metano variando el flujo total de entrada (1.0 a 5.0 litros estándar por minuto, SLPM) y la masa de óxido (0.66 a 3.33 gramos).

Para eliminar los efectos difusionales entre la superficie y el interior de las partículas (limitaciones internas), se suministró un flujo de $3.0 \mathrm{SLPM}$ a $650^{\circ} \mathrm{C}$ con $2.5 \% \mathrm{v} / \mathrm{v}$ de metano, para 2.0 gramos de material activo con diámetros de partícula entre 1.2 y $2.8 \mathrm{~mm}$.

\section{Dependencia de la velocidad de reacción respecto a las concentraciones de metano y oxígeno}

Para operar el reactor de manera diferencial se mantuvo la conversión de metano inferior a $15 \%$, (Fogler, 2001; Hayes y Kolaczkowski, 1997; Froment y Bischoff, 1990). Para determinar el orden de reacción respecto al metano, se varió la concentración de éste con $100 \%$ de oxígeno en exceso, para flujo de mezcla reactiva de 3 SLPM, sin suministro de nitrógeno. Las concentraciones de metano en la entrada fueron $1.5,2.0,2.5$ y $3.0 \% \mathrm{v} / \mathrm{v}$ ajustadas en línea por medio del analizador NDIR a temperaturas entre 600 y $650^{\circ} \mathrm{C}$, con incrementos de $12.5^{\circ} \mathrm{C}$. El lecho se empacó con partículas de diámetro entre 1.6 y $2.1 \mathrm{~mm}$.

Para obtener el orden de reacción respecto al oxígeno, se midieron los productos de combustión manteniendo la concentración de metano constante $(3.0 \% \mathrm{v} / \mathrm{v})$ para un flujo de mezcla reactiva de 3 SLPM con concentraciones de oxígeno $6.0,7.0,8.0$ y $9.0 \% \mathrm{v} / \mathrm{v}$, correspondientes a la relación estequiométrica, 17, 33 y 50\% en exceso respectivamente, con diámetros de partículas entre 1.6 y $2.1 \mathrm{~mm}$. El aire se suministró diluido con nitrógeno y se ajustaron las concentraciones en línea con el analizador de gases NDIR.

\section{Dependencia de la velocidad de reacción respecto a la temperatura}

Para una composición constante de mezcla gaseosa reactiva $\left(2.5 \% \mathrm{v} / \mathrm{v} \mathrm{CH}_{4}\right.$ y $\left.10.0 \% \mathrm{v} / \mathrm{v} \mathrm{O}_{2}\right)$ y variando la temperatura de entrada al lecho entre 600 y $650^{\circ} \mathrm{C}$, con incrementos de $12.5^{\circ} \mathrm{C}$, se midió 
la concentración de los gases de salida $\left(\mathrm{CH}_{4}, \mathrm{CO}, \mathrm{CO}_{2}\right.$ y $\left.\mathrm{O}_{2}\right)$. La velocidad de reacción se determinó a partir de las concentraciones de metano a la entrada y a la salida del reactor, manteniendo la conversión de metano inferior al $15 \%$ con el fin de garantizar comportamiento de reactor diferencial. La conversión de metano se calculó como la relación porcentual entre las moles de metano que reaccionaron y las alimentadas al reactor.

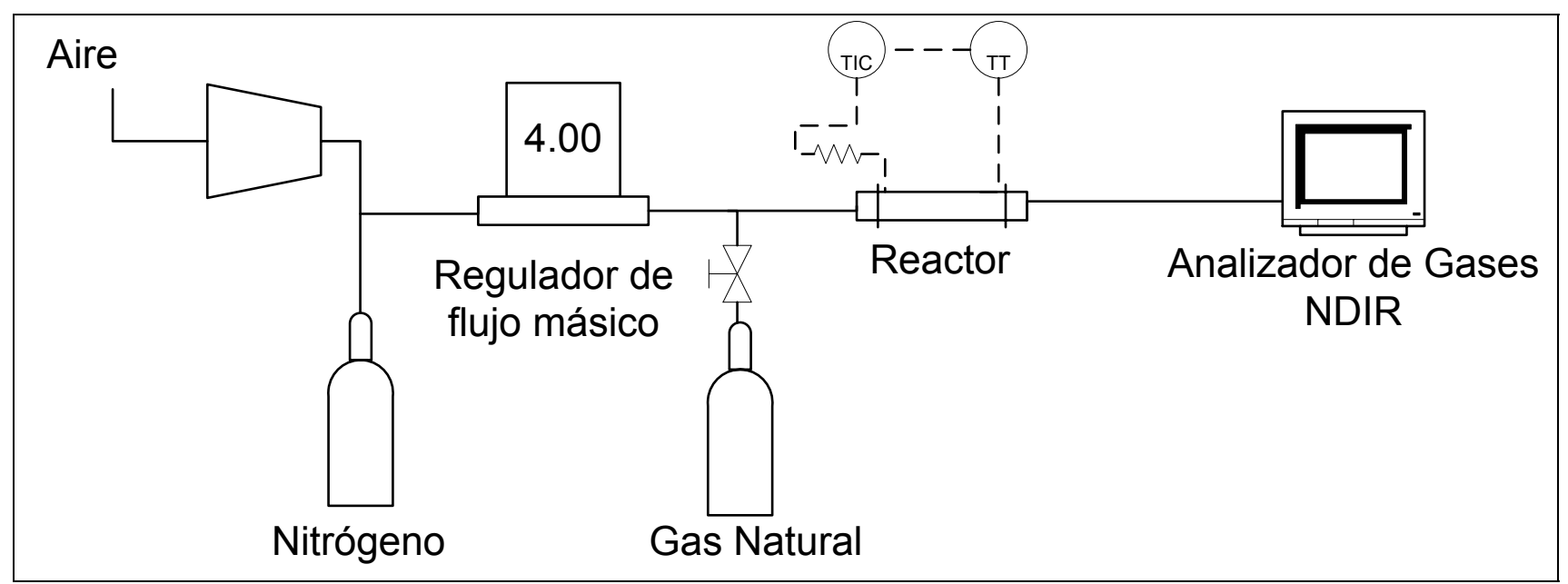

Fig. 1: Esquema del montaje experimental para variar la concentración de metano y oxígeno en la mezcla reactiva.

\section{RESULTADOS Y DISCUSIÓN}

\section{Eliminación de las limitaciones de transporte}

La medición de la velocidad de consumo de metano (mol/g min) para mezclas aire-gas natural $(2.5 \%$ $\mathrm{v} / \mathrm{v}$ de metano), con relación peso de óxido a flujo de reactivos $\left(\mathrm{W} / \mathrm{F}_{\mathrm{o}}\right.$ ) de $0.04 \mathrm{~g} \mathrm{~s} / \mathrm{mL}$, dió como resultado que para flujos superiores a 2 SLPM, la velocidad de reacción se mantuvo constante en $0.018 \mathrm{~mol} / \mathrm{g} \mathrm{min}$, lo cual es indicativo de la no existencia de limitaciones de transporte entre el seno del gas y la superficie de la partícula.

Para determinar las condiciones que eliminaran las limitaciones difusionales internas, se alimentaron al reactor empacado con 2 gramos de sólido activo, 3 SLPM de mezcla aire-gas natural a $650^{\circ} \mathrm{C}$, $2.5 \% \mathrm{v} / \mathrm{v}$ de metano y se midió su velocidad de consumo $\left(-\mathrm{r}_{\mathrm{CH} 4}\right)$ en función del diámetro de partícula $\mathrm{D}_{\mathrm{p}}$ correspondientes a $1.19,1.41,1.65,1.98,2.38$ y $3.33 \mathrm{~mm}$. De la curva $-r_{C H 4}$ versus $D_{p}$ se obtuvo que para tamaños menores a $2.18 \mathrm{~mm}$, la velocidad de reacción no depende de esta variable, indicando que no hay efectos de difusión interna cuando el lecho se empaca con partículas de diámetro inferior. Esto se corroboró con el coeficiente de Weisz-Prater (Fogler, 2001), $\mathrm{C}_{\mathrm{WP}}=0.037$, que al ser mucho menor que la unidad, indica la ausencia de limitaciones difusionales internas para $-r_{\mathrm{CH} 4}=3.5 \mathrm{E}-4 \mathrm{~mol} /(\mathrm{g} \mathrm{min}), \rho_{\mathrm{C}}=0.94 \mathrm{~g} / \mathrm{cm}^{3}, \mathrm{R}_{\mathrm{p}}=0.11 \mathrm{~cm}, D_{\mathrm{e}}=1.59 \mathrm{~cm}^{2} / \mathrm{s}, \mathrm{C}_{\mathrm{CH} 4, \mathrm{~s}}=1.12 \mathrm{e}-6 \mathrm{~mol} / \mathrm{cm}^{3}$.

Con base en los anteriores hallazgos, la determinación de los parámetros cinéticos se realizó con flujos superiores a 2 SLPM y diámetros de partícula menores a $2.18 \mathrm{~mm}$, con el fin de evitar reacciones controladas por efectos difusionales.

\section{Dependencia de la velocidad de reacción respecto a la concentración de metano}

La Figura 2 muestra la velocidad de consumo de metano $\left(-\mathrm{r}_{\mathrm{CH}}\right.$, $\left.\mathrm{mol} / \mathrm{g} \mathrm{min}\right)$ en función de su concentración molar $\left(\mathrm{C}_{\mathrm{CH} 4}, \mathrm{~mol} / \mathrm{L}\right)$ en la mezcla reactiva, para temperaturas de alimentación entre 600 y $650^{\circ} \mathrm{C}$ con incrementos de $12.5^{\circ} \mathrm{C}$. Se evidencia la limitación de $-\mathrm{r}_{\mathrm{CH} 4}$ por saturación superficial, dado que en $8.93 \times 10^{-4} \mathrm{~mol}$ de $\mathrm{CH}_{4}$ por litro, la velocidad de consumo de metano cambia de pendiente positiva a negativa. Este comportamiento indica que la velocidad de reacción no se ajusta a una ley 
de potencias del tipo $-r_{\mathrm{CH}_{4}}=k \cdot C_{\mathrm{CH}_{4}}^{n} \cdot C_{\mathrm{O}_{2}}^{m}$, donde $k$ es la constante cinética, $C_{\mathrm{CH}_{4}}$ y $C_{\mathrm{O}_{2}}$ son las concentraciones molares de metano y oxígeno, respectivamente.

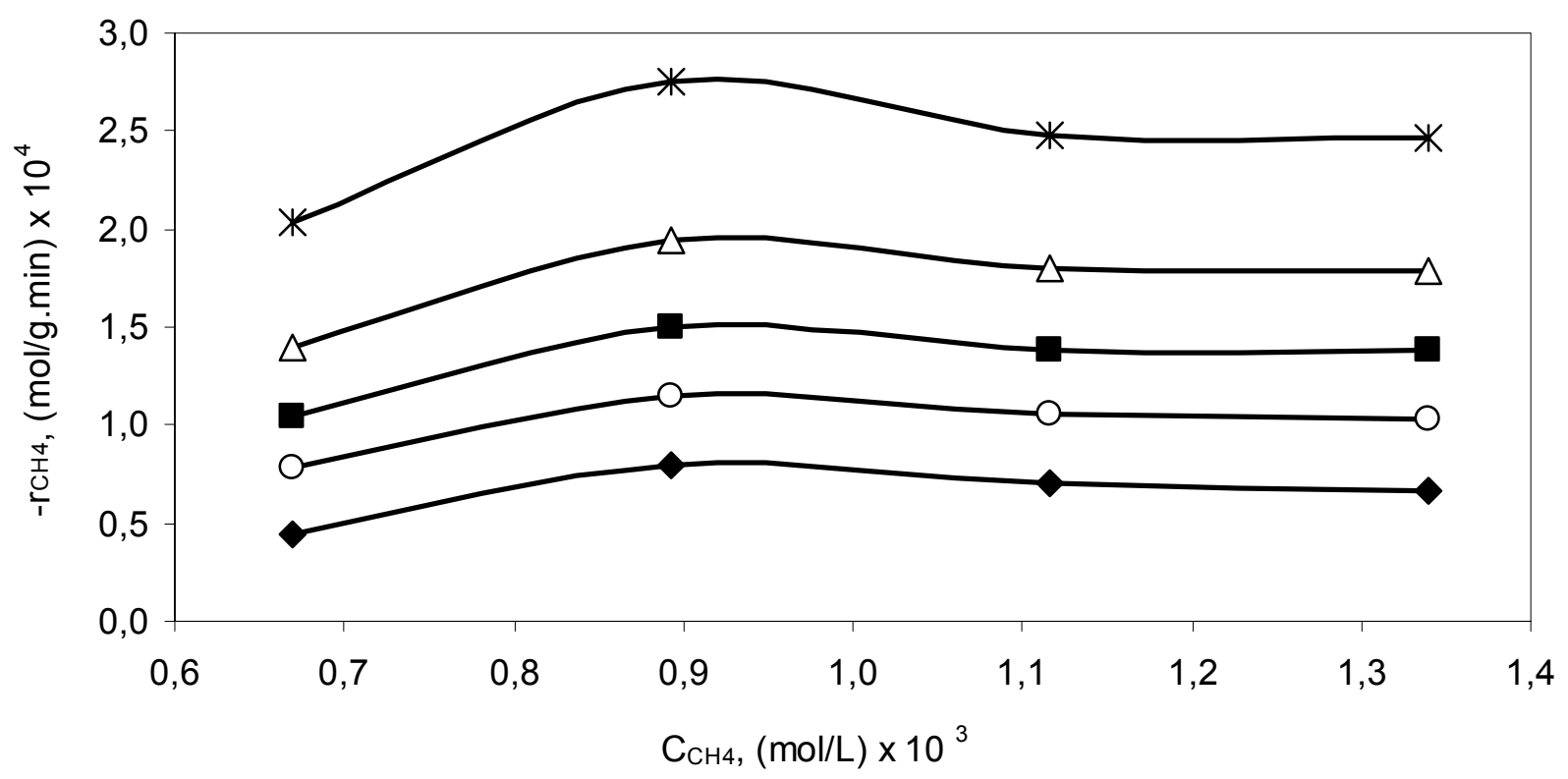

Fig. 2: Variación de la velocidad de reacción con la concentración de metano a diferentes temperaturas, $600^{\circ} \mathrm{C}, 0612.5^{\circ} \mathrm{C}, 625^{\circ} \mathrm{C}, \nabla 637.5^{\circ} \mathrm{C}, * 650^{\circ} \mathrm{C}$.

En combustión de hidrocarburos gaseosos promovida por una superficie sólida usualmente la cinética es del tipo Langmuir-Hinshelwood-Hougen-Watson, LHHW (Hayes y Kolaczkowski, 1997). El mecanismo de reacción tipo LHHW más aceptado para la oxidación de metano con oxígeno (Hayes y Kolaczkowski, 1997; Berg y Järas, 1994), sigue las etapas indicadas por las ecuaciones (1) a (4), donde $S_{1}$ y $S_{2}$ son sitios de adsorción diferentes sobre la superficie del sólido: (1) adsorción no disociativa de metano en $S_{1}$, (2) adsorción no disociativa de oxígeno en $S_{2}$, (3) reacción superficial entre metano y oxígeno adsorbidos y (4) desorción de radicales libres, los cuales completan su oxidación en fase gaseosa hasta $\mathrm{CO}_{2}$ y $\mathrm{H}_{2} \mathrm{O}$ dejando libres los sitios activos $\mathrm{S}_{1}$ y $\mathrm{S}_{2}$.

$$
\begin{aligned}
& \mathrm{CH}_{4(\mathrm{~g})}+\mathrm{S}_{1} \Leftrightarrow \mathrm{CH}_{4} \cdot \mathrm{S}_{1} \\
& \mathrm{O}_{2(\mathrm{~g})}+\mathrm{S}_{2} \Leftrightarrow \mathrm{O}_{2} \cdot \mathrm{S}_{2} \\
& \mathrm{CH}_{4} \cdot \mathrm{S}_{1}+\mathrm{O}_{2} \cdot \mathrm{S}_{2} \Leftrightarrow \mathrm{CH}_{3} \cdot \mathrm{S}_{1}+\mathrm{HO}_{2} \cdot \mathrm{S}_{2} \\
& \mathrm{CH}_{3} \cdot \mathrm{S}_{1}+\mathrm{HO}_{2} \cdot \mathrm{S}_{2} \Leftrightarrow \mathrm{CH}_{3} \cdot \mathrm{S}_{1}+\mathrm{HO}_{2} \cdot \mathrm{S}_{2}
\end{aligned}
$$

El mecanismo representado por las ecuaciones (1) a (4) lleva a la expresión (5), con $k$ constante cinética, $K_{\mathrm{CH} 4}$ y $\mathrm{K}_{02}$, constantes de equilibrio de adsorción de metano y oxígeno; $\mathrm{C}_{\mathrm{CH} 4}$ y $\mathrm{C}_{02}$, concentraciones molares de metano y oxígeno, respectivamente; $\theta_{\mathrm{CH} 4}$ y $\theta_{\mathrm{O} 2}$, cubrimientos superficiales de metano y oxigeno.

$$
-r_{\mathrm{CH}_{4}}=k \cdot\left[\frac{\mathrm{K}_{2} \cdot \mathrm{C}_{2}}{1+\mathrm{K}_{\mathrm{O}_{2}} \cdot \mathrm{C}_{\mathrm{O}_{2}}}\right] \cdot\left[\frac{{ }_{\mathrm{CH}_{4}} \cdot{ }^{\mathrm{CH}_{4}}}{1+\mathrm{K}_{\mathrm{CH}_{4}} \cdot{ }^{-C_{\mathrm{CH}_{4}}}}\right]=k \cdot \Theta_{\mathrm{O}_{2}} \cdot \Theta_{\mathrm{CH}_{4}}
$$

Debido al alto exceso de oxigeno utilizado (100\%), la velocidad de reacción puede considerarse independiente de su concentración. Si en (5) se sustituye $k \cdot\left[K_{02} \cdot C_{\mathrm{O} 2} /\left(1+K_{02} \cdot C_{\mathrm{O} 2}\right)\right]$ por $k_{s}$ ", se obtiene 
la ecuación (6), que muestra la velocidad de consumo de metano $\left(-\mathrm{r}_{\mathrm{CH} 4}\right)$ en función únicamente de la constante de equilibrio de adsorción $\left(\mathrm{K}_{\mathrm{CH} 4}\right)$, concentración de metano $\left(\mathrm{C}_{\mathrm{CH} 4}\right)$ y $k_{s}$ " que es el producto de la constante cinética $(k)$ y el cubrimiento superficial de oxígeno, $\Theta_{\mathrm{CH}_{4}}$.

$$
-r_{\mathrm{CH}_{4}}=k_{s}{ }^{\prime \prime} \frac{{ }_{\mathrm{CH}_{4}}{ }^{C_{\mathrm{CH}_{4}}}}{1+\mathrm{K}_{\mathrm{CH} 4}{ }^{C} \mathrm{CH}_{4}}
$$

La ecuación (7) corresponde a la linealización de la velocidad de consumo de metano dada por (6)

$$
\frac{{ }_{\mathrm{CH}_{4}}}{-r_{\mathrm{CH}_{4}}}=\frac{1}{k_{s}{ }^{\prime \prime} \cdot \mathrm{K}_{\mathrm{CH}_{4}}}+\frac{{ }_{\mathrm{CH}_{4}}}{k_{s}{ }^{\prime \prime}}
$$

La Figura 3 muestra el ajuste de los resultados experimentales al comportamiento que predice la ecuación (7). Se observa que el coeficiente de correlación $\mathrm{R}^{2}$ es superior a 0.8 para temperatura entre 612.5 y $650^{\circ} \mathrm{C}$, confirmándose que para dicho intervalo la reacción sigue un mecanismo de adsorción en sitios distintos con limitaciones por reacción superficial donde el orden de reacción para el metano es aproximadamente uno. Este resultado concuerda con los obtenidos por Berg y Järas (1994) para combustión de metano con oxígeno sobre MgO puro y por Roos et al. (1989) para la oxidación parcial de metano en aire sobre $\mathrm{Li} / \mathrm{MgO}$. A $600^{\circ} \mathrm{C}$ se tiene una alta dispersión de los datos reflejada por un coeficiente de correlación de 0.511 , lo cual indica que para esta temperatura el modelo planteado no se ajusta a los resultados experimentales; lo cual puede deberse a que el consumo de metano está limitado a las reacciones en la superficie del material, mientras a temperaturas superiores hay una contribución considerable de la oxidación en fase homogénea.

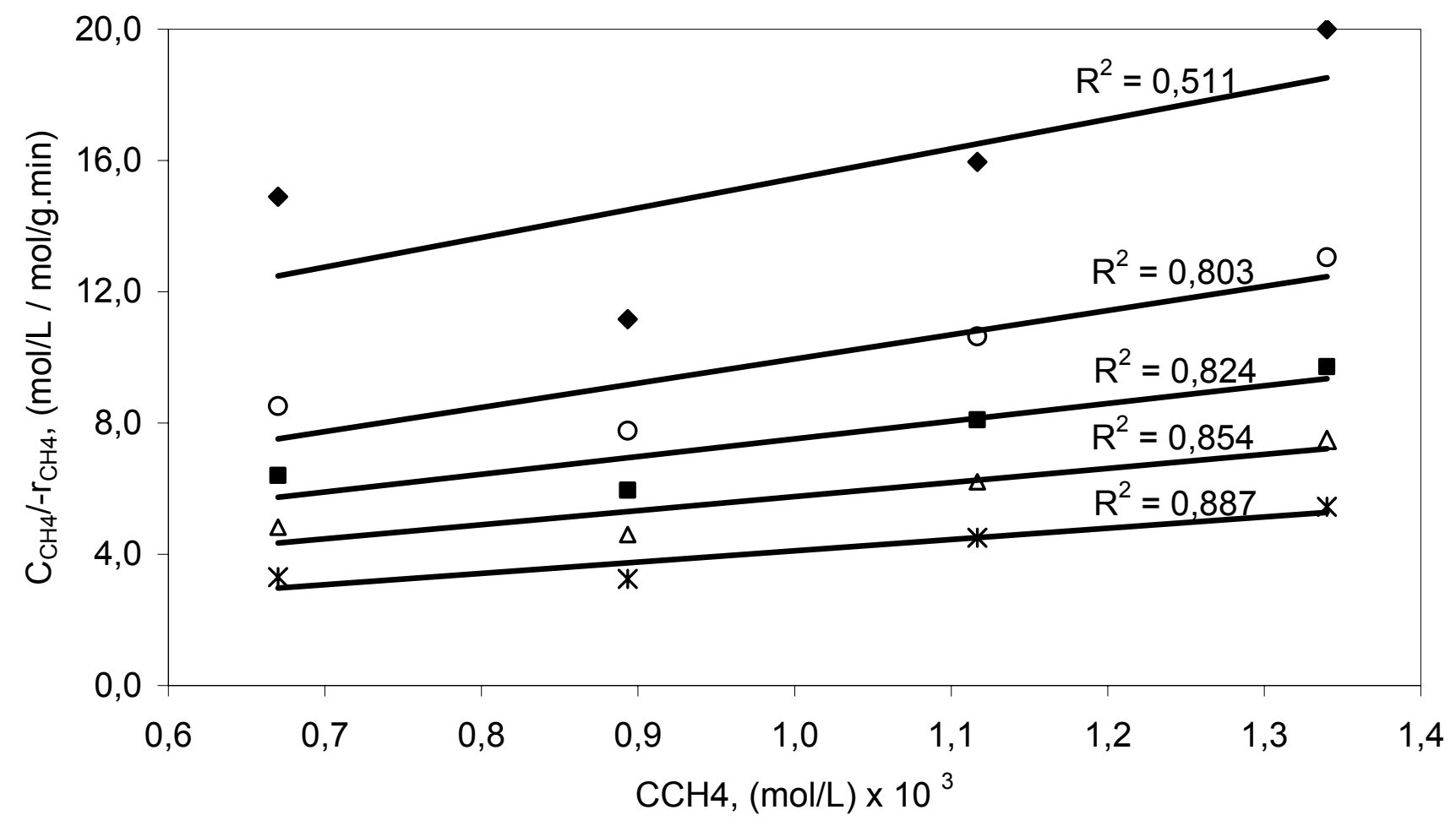

Fig. 3: Concentración de metano versus concentración de metano / velocidad de reacción. $\bullet 600^{\circ} \mathrm{C}$, o $612.5^{\circ} \mathrm{C}, 625^{\circ} \mathrm{C}, \nabla 637.5^{\circ} \mathrm{C}, * 650^{\circ} \mathrm{C}$ 


\section{Dependencia de la velocidad de reacción respecto a la concentración de oxígeno}

Para concentración de metano constante $(3.0 \% \mathrm{v} / \mathrm{v})$ y temperaturas de mezcla reactiva entre 600 y $650^{\circ} \mathrm{C}$ con incrementos de $12.5^{\circ} \mathrm{C}$, se obtuvo muy baja conversión para las diferentes concentraciones de oxígeno en la mezcla reactiva $(6.0,7.0,8.0$ y $9.0 \% \mathrm{v} / \mathrm{v})$. Hubo una leve incidencia de la concentración de oxígeno sobre la conversión de metano, hasta aproximadamente el 8,0\% v/v en $\mathrm{O}_{2}$; a partir de esta composición, se reduce aún más la influencia del oxígeno. Este comportamiento permite afirmar que para los excesos de oxígeno evaluados, es despreciable la dependencia de la velocidad de reacción con respecto a la concentración de este reactivo y por tanto la reacción es de orden cero respecto al oxígeno, tal como lo describe la ecuación (6). Este resultado concuerda con el que reportan Choudhary et al. (2002) para combustión de metano con exceso de oxígeno sobre catalizadores de platino, pero difiere de lo obtenido por Berg y Järas (1994) para combustión de metano con oxígeno sobre $\mathrm{MgO}$ puro. Igualmente varía respecto a los obtenidos por Roos et al. (1989) e Ito et al. (1985) en la oxidación parcial de metano sobre Li/MgO, los cuales encontraron orden de reacción para el oxígeno diferente de cero, usando relaciones oxígeno a metano menores a las del presente trabajo.

\section{Dependencia de la velocidad de reacción respecto a la temperatura}

El efecto de la temperatura se manifiesta en la constante de equilibrio de adsorción del metano $\left(K_{\mathrm{CH}}\right)$ y en la constante global $k_{s}$ " de la ecuación (6). Con las pendientes e interceptos de las rectas de la Figura 3 se obtienen los valores de $K_{C H 4}$ y $k_{s}$ " de la Tabla 1 , donde se aprecia la influencia de la temperatura sobre la constante de equilibrio de adsorción del metano $\left(K_{\mathrm{CH}}\right)$, la cual muestra una marcada dependencia evidenciada al cuadruplicarse el valor de la constante ante un incremento de $50^{\circ} \mathrm{C}$ en la temperatura.

Tabla 1: Constantes de equilibrio de adsorción y de cinética global.

\begin{tabular}{|c|c|c|c|c|}
\hline $\begin{array}{c}\text { Temperatura } \\
\left({ }^{\circ} \mathrm{C}\right)\end{array}$ & $\begin{array}{c}\text { Pendiente }\left(1 / k_{s}{ }^{\prime \prime}\right) \\
(\mathrm{g} \mathrm{min} / \mathrm{mol}) \times 10^{-3}\end{array}$ & $\begin{array}{c}k_{s}{ }^{\prime \prime} \\
(\mathrm{mol} / \mathrm{g} \mathrm{min}) \times 10^{4}\end{array}$ & $\begin{array}{c}\text { Intercepto }\left(1 / k_{s}{ }^{\prime} K_{\mathrm{CH} 4}\right) \\
1 / \mathrm{mol} / \mathrm{g} \mathrm{min} \mathrm{mol} / \mathrm{L}\end{array}$ & $\begin{array}{c}K_{\mathrm{CH} 4} \\
(\mathrm{~L} / \mathrm{mol}) \times 10^{-3}\end{array}$ \\
\hline 600.0 & 9.009 & 1.110 & 6.449 & 1.397 \\
\hline 612.5 & 7.375 & 1.356 & 2.580 & 2.858 \\
\hline 625.0 & 5.396 & 1.853 & 2.118 & 2.548 \\
\hline 637.5 & 4.297 & 2.327 & 1.460 & 2.943 \\
\hline 650.0 & 3.445 & 2.903 & 0.662 & 5.204 \\
\hline
\end{tabular}

La ecuación de Arrhenius indica la dependencia exponencial de la constante cinética con temperatura, $k=A \exp \left(-E_{a} / R T\right)$, donde $A$ es el factor preexponencial, $E_{a}$ la energía de activación, $R$ la constante universal de los gases y $T$ la temperatura absoluta. Al reemplazar $k$ en la constante global $k_{s}$, se obtiene la ecuación (8)

$$
k_{s}{ }^{\prime \prime}=A \cdot \exp \left(-E_{a} / R T\right) \cdot\left[\frac{K_{O_{2}} \cdot C_{O_{2}}}{1+K_{O_{2}} \cdot C_{O_{2}}}\right]
$$

En la ecuación (8) se sustituye $A^{\prime}$ por $A \cdot\left[K_{02} C_{02} /\left(1+K_{02} C_{O 2}\right)\right]$, asumiendo que es despreciable la dependencia del cubrimiento superficial del oxígeno respecto a la temperatura, dado el exceso de oxigeno usado. Al aplicar logaritmo natural a ambos lados de la ecuación (8) modificada, resulta la ecuación (9).

$$
\ln \left(k_{S}^{\prime \prime}\right)=\frac{-E_{a}}{R T}+\ln \left(A^{\prime}\right)
$$

La ecuación (9) se representa gráficamente en la Figura 4, en la cual no se tuvo en cuenta el valor de $k_{s}$ "correspondiente a $600^{\circ} \mathrm{C}$ dada la pobreza del ajuste de los datos experimentales con el modelo 
cinético propuesto. De la ecuación de Arrhenius linealizada (9) se determinó a partir de la pendiente, la energía de activación $E_{a}=136.64 \mathrm{~kJ} / \mathrm{mol}(32.66 \mathrm{kcal} / \mathrm{mol})$ y del intercepto, el factor preexponencial $A^{\prime}=15946 \mathrm{~mol} / \mathrm{g} \mathrm{min}$.

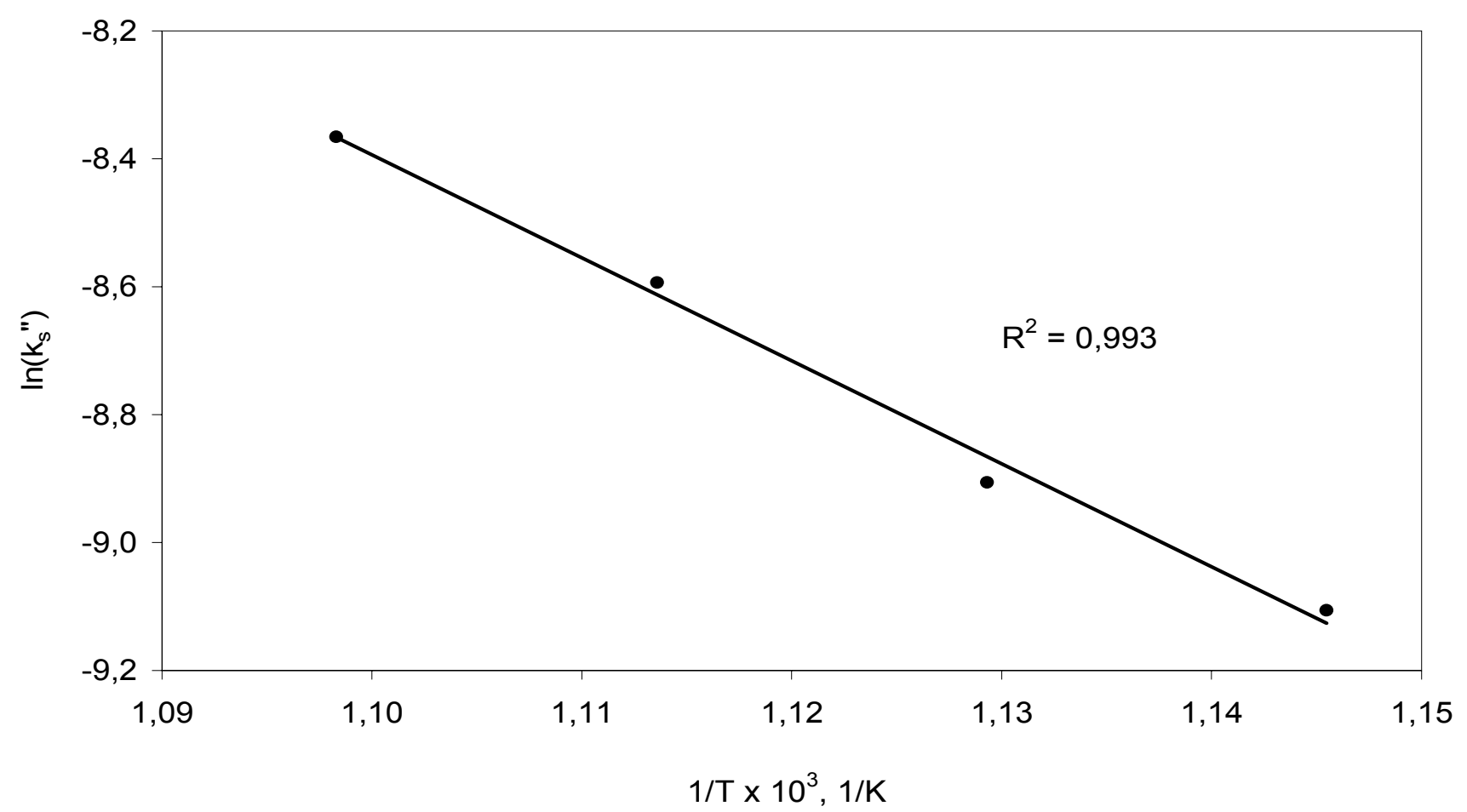

Fig. 4: Linealización de la ecuación de Arrhenius para combustión de metano sobre CaO/MgO.

La energía de activación aquí obtenida $(136.64 \mathrm{~kJ} / \mathrm{mol})$ es $46.85 \%$ menor que la requerida para combustión térmica $(257.07 \mathrm{~kJ} / \mathrm{mol})$ reportada por Arai et al. (1986) y $5.11 \%$ mayor que la determinada por Berg y Jaras (1994) para combustión de metano sobre MgO puro (130.00 kJ/mol). También supera los valores reportados por Arai et al. (1986) en $13.50 \%$ para perovskitas base lantano $(120.384 \mathrm{~kJ} / \mathrm{mol})$ y en $18.44 \%$ para platino soportado en alúmina $(115.368 \mathrm{~kJ} / \mathrm{mol})$.

Este resultado muestra que el $\mathrm{CaO} / \mathrm{MgO}$ es activo para la combustión de gas natural con aire, y aunque no alcanza las actividades de los metales nobles ni de las perovskitas, su elevada estabilidad térmica lo hace apto para aplicaciones de combustión sin llama donde se requieran temperaturas que superen los $800^{\circ} \mathrm{C}$ (Gómez et al., 2008; Gómez et al., 2007). Cabe anotar que los precursores del material activo utilizado en este trabajo son de bajo costo y el método de preparación sencillo y económico. La actividad del material probado posiblemente está influenciada por la presencia del calcio en la superficie de las partículas de óxido de magnesio, promoviendo la producción de radicales libres que terminan de oxidarse en la fase gaseosa (Zwinkels et al., 1993; Pfefferle y Pfefferle, 1987).

Al reemplazar $A^{\prime}$ y $E_{a}$ en la ecuación (8) y llevar el resultado junto con el promedio de $K_{C H 4}$ entre 612.5 y $650^{\circ} \mathrm{C}$ a la ecuación (6), la cinética para el consumo de metano en combustión de gas natural sobre $\mathrm{CaO} / \mathrm{MgO}$, queda expresada por la ecuación (10); donde $-r_{\mathrm{CH} 4}$ está en $\mathrm{mol} / \mathrm{g}$ min, $T$ en Kelvin, $\mathrm{C}_{\mathrm{CH} 4}$ en $\mathrm{mol} / \mathrm{L}$ y R $=8.314 \mathrm{~J} / \mathrm{mol} \mathrm{K}$.

$$
-r_{\mathrm{CH}_{4}}=15946 \cdot \exp (-136640 / R \cdot T)\left(\frac{3388 \cdot C_{C H_{4}}}{1+3388 \cdot C_{C H_{4}}}\right)
$$

La ecuación (10), no reportada en trabajos previos, expresa la velocidad de consumo de metano para combustión de mezclas gas natural - aire en exceso de oxígeno sobre $\mathrm{CaO} / \mathrm{MgO}$, en términos de la temperatura absoluta y la concentración de metano. Al experimentar con gas natural libre $(98 \%$ 
$\left.\mathrm{CH}_{4}\right)$ con un porcentaje muy bajo de etano y propano $(0.25 \%$ y $0.05 \%$, respectivamente), la cinética global la determina fundamentalmente el metano; tal como se evidenció al comparar lo obtenido en el presente trabajo con resultados para metano de alta pureza (Gómez et. al., 2008).

\section{CONCLUSIONES}

Los estudios cinéticos realizados permiten concluir que la reacción de combustión sin llama de gas natural en exceso de oxígeno sobre $\mathrm{CaO} / \mathrm{MgO}$, presenta una cinética tipo LHHW con reacción superficial como etapa controlante, siguiendo un mecanismo de sitio dual con adsorción de metano y oxígeno no disociativa.

Se obtuvieron ordenes de reacción uno para el metano y aproximadamente cero para el oxígeno en combustión sin llama de gas natural con aire sobre $\mathrm{CaO} / \mathrm{MgO}$, operando con concentraciones de metano menores al límite inferior de inflamabilidad en combustión térmica $\left(\begin{array}{llll}5.0 \% & \mathrm{CH}_{4}\end{array}\right)$ y concentraciones de oxígeno desde la estequiométrica hasta $100 \%$ de exceso.

$\mathrm{El} \mathrm{CaO} / \mathrm{MgO}$ que se preparó es activo en combustión sin llama de gas natural con aire, bajo condiciones de exceso de oxígeno, lo cual se evidencia con la menor energía de activación requerida si se compara con la de combustión térmica. Dicho hallazgo permite trascender su uso no solo para soportar metales nobles sino también como material activo de fácil preparación, bajo costo y alta estabilidad térmica.

La expresión cinética obtenida es fundamental para el diseño de sistemas de combustión sin llama de gas natural y dispositivos de depuración de emisiones gaseosas que contengan metano en concentraciones menores al límite inferior de inflamabilidad, operando a temperaturas superiores a las que permiten los metales nobles y las perovskitas e inferiores a las que se forman los NOx térmicos.

\section{AGRADECIMIENTOS}

A la Universidad de Antioquia (Comité para el Desarrollo de la Investigación, CODI) por la financiación. Al Grupo Ciencia y Tecnología del Gas y URE por el apoyo logístico.

\section{NOMENCLATURA}

\section{Símbolos}

A: $\quad$ factor preexponencial de la expresión de Arrhenius

A': $\quad$ producto del factor preexponencial de Arrhenius y el cubrimiento superficial de oxígeno

$\mathrm{C}_{\mathrm{CH} 4}$ : concentración molar de metano

$\mathrm{C}_{\mathrm{O} 2}: \quad$ concentración molar de oxígeno

$\mathrm{C}_{\mathrm{WP}}$ : coeficiente de Weisz-Prater

$D_{e}: \quad$ difusividad efectiva

$\mathrm{E}_{\mathrm{a}}$ : $\quad$ energía de activación

$\mathrm{k}$ : $\quad$ constante cinética según la expresión de Arrhenius

$\mathrm{K}_{\mathrm{CH} 4}$ : constante de equilibrio de adsorción de metano

$\mathrm{K}_{\mathrm{O} 2}: \quad$ constante de equilibrio de adsorción de oxígeno

$k_{s}{ }^{\prime \prime}$ : $\quad$ producto de la constante cinética y el cubrimiento superficial de oxígeno

R: $\quad$ constante universal de los gases

Rp: $\quad$ radio de partícula de catalizador

$-\mathrm{CH}_{4}$ : velocidad de consuno de metano

T: temperatura absoluta

Símbolos griegos

$\Theta_{\mathrm{CH}_{4}}: \quad$ cubrimiento superficial de metano 


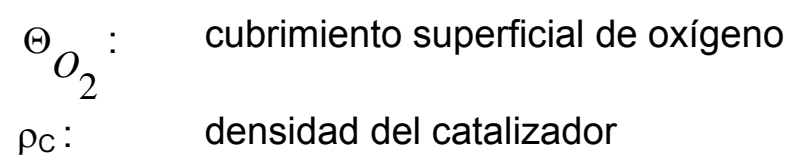

\section{Subíndices}

(g): $\quad$ estado gaseoso

S: $\quad$ superficial

\section{REFERENCIAS}

Aigler, J.M. y J.H. Lunnsford; Oxidative Dimerization of Methane Over MgO and Li+/MgO Monoliths, Appl. Catal.: 70(1), 29-42 (1991).

Arai, H. y otros tres autores; Catalytic Combustion of Methane Over Various Perovskite-type Oxides, Appl. Catal.: 26, 265-276 (1986).

Baylet, A., y otros cuatro autores; High Catalytic Activity and Stability of Pd Doped Hexaaluminate Catalysts for the $\mathrm{CH}_{4}$ Catalytic Combustión, Appl. Catal. B: Environmental: 77(3-4), 237-247 (2008).

Berg, M. y S. Järas; Catalytic Combustion of Methane Over Magnesium Oxide, Appl. Catal. A: General: 114, 227-241 (1994).

Berg, M. y S Järas; High Temperature Stable Magnesium Catalyst for Catalytic Combustion of Methane: a Comparison with Manganese-substututed Barium Hexaaluminate, Catal. Today: 26, 223229 (1995).

Campagnoli, E. y otros seis autores; Effect of Preparation Method on Activity and Stability of LaMnO $\mathrm{O}_{3}$ and $\mathrm{LaCoO}_{3}$ Catalysts for the Flameless Combustion of Methane, Appl. Catal. B: Environmental: 55(2), 133-139 (2005).

Chen, M. y otros cuatro autores; Synthesis of Nanoparticle Ce-Mg-O Mixed Oxide as Efficient Support for Mthane Oxidation, J. Molec. Catal. A: Chemical: 237(1-2), 132-136 (2005).

Chiarello, G. L. y otros cuatro autores; Preparation by Flame Spray Pyrolysis of $A B O_{3 \pm \delta}$ Catalysts for the Flameless Combustion of Methane, Catal. Today: 117(4), 549-553 (2006).

Choudhary, T.V.; V.R. Banerjee y V.R. Choudhary; Catalyst for Combustion of Methane and Lower Alkanes, Appl. Catal. A: General: 234, 1-23 (2002).

Cunnigham. J.C. Healy, D. McNamara, S. O'Brien; Preparation and Characterization of MgO Powders Having $\mathrm{Ca}^{2+}$ or $\mathrm{Ba}^{2+}$ as Surface Dopants, Catal. Today: 2, 557-567 (1988).

Dubois J.L. y C J. Cameron; Common Features of Oxidative Coupling of Methane Cofeed Catalyst, App. Catal.: 67, 49-71 (1990).

Ersson, A., K. Persson, I.K. Adu y S.G. Järas; A Comparison Between Hexaaluminates and Perovskites for Catalytic Combustion Applications, Catal. Today: 112(1-4), 157-160 (2006).

Fogler, H.S.; Elementos de Ingeniería de las Reacciones Químicas, $3^{a}$ edición, Pearson educación, México, (2001).

Froment, G.F. y K.B. Bischoff; Chemical Reactor Analysis and Design, 2a edición, J. Wiley, New York, USA (1990).

Gluhoi, A.G., y B.E. Nieuwenhuys; Catalytic oxidation of saturated hydrocarbons on multicomponent $\mathrm{Au} / \mathrm{Al}_{2} \mathrm{O}_{3}$ catalysts: Effect of various promoters, Catal. Today: 119(1-4), 305-310 (2007). 
Gómez, E., M. Sánchez y A. Jaramillo; Combustión sin Llama de Mezclas Pobres Metano-Aire sobre MgO Adicionado con CaO, Información Tecnológica: 19(6), diciembre de 2008. En Prensa.

Gómez, E., M. Sánchez y A. Jaramillo; Combustión Sin Llama de Gas Natural sobre Lecho Fijo de Óxido de Magnesio, Dyna: 74(151), 107-114 (2007).

Gómez, E., Jaramillo, A., Múnera, J. y M. Sánchez; Preparación, Caracterización y Evaluación de MgO para Combustión Sin Llama de Gas Natural, Revista Facultad de Ingeniería: (37), 41-49 (2006).

Hayes, R.E. y S.T. Kolaczkowski; Introduction to Catalytic Combustion, Gordon and Breach Science Publishers, Amsterdam (1997).

Ito, T., y otros tres autores; Oxidative Dimerization of Methane Over Lithium-Promoted Magnesium Oxide Catalyst, J. Am. Chem. Soc.: 107, 50-62 (1985).

Li, S. y X. Wang; Catalytic Combustion of Methane Over Mn-Substituted Ba-La-Hexaaluminate Nanoparticles, J. Alloy Compd.: 432(1-2), 333-337(2007).

Kimble, J.B. y J.H. Kolts; Playing Matchmaker with Methane, Chemtech: 501-505 (1987).

McCune R.C. y P. Wynblatt; Calcium Segregation to a Magnesium Oxide (100) Surface, J. Am. Ceram. Soc.: 66(2), 111-116 (1983).

Persson, K., K. Jansson y S.G. Järas; Characterisation and Microstructure of Pd and Bimetallic Pd-Pt Catalysts During Methane Oxidation, J. Catal.: 245(2), 401-414 (2007).

Petrovic, S. y otros ocho autores; $\mathrm{LaMO}_{3}(\mathrm{M}=\mathrm{Mg}$, Ti, Fe) Perovskite Type Oxides: Preparation, Characterization and Catalytic Properties in Methane Deep Oxidation, Appl. Catal. B: Environmental, 79(2), 186-198 (2008).

Pfefferle, L.D. y W.C. Pfefferle; Catalysis in Combustion, Catal. Rev. Sci. Eng.: 29, 219-267 (1987).

Ramadj, O.M. y otros tres autores; Catalytic Combustion of Methane over High Copper-Loading ZSM5 Catalysts, J. Natural Gas Chemistry: 16(3), 258-265 (2007).

Ren, X., y otros tres autores; Catalytic Properties of Fe and Mn Modified Lanthanum Hexaaluminates for Catalytic Combustion of Methane, Catal. Commun.: (2007), doi:10.1016/j.catcom.2007.09.004.

Requies, J. y otros ocho autores; Palladium-Manganese Catalysts Supported on Monolith Systems for Methane Combustión, Appl. Catal. B: Environmental: 79(2), 122-131 (2008).

Roos, J. A y otros cuatro autores; Kinetic and Mechanistic Aspects of the Oxidative Coupling of Methane Over Li/MgO, Appl. Catal.: 52(1), 131-145 (1989).

Spretz, R. y otros tres autores; Fe/MgO Formulations for the Catalytic Combustion of Methane, J. Catal.: 194, 167-174 (2000).

Teng, F. y otros ocho autores; Effect of the Flowing Gases of Steam and $\mathrm{CO}_{2}$ on the Texture and Catalytic Activity for Methane Combustion of MgO Powders, Micropor. Mesopor. Mater. oi:10.1016/j.micromeso.2007.07.013, (2007).

Ulla, M. A., y otros cuatro autores; Catalytic Combustión of Methane on Co/MgO: Characterization of Active Cobalt Sites, Appl. Catal. B: Environmental: 29, 217-229 (2001).

Yoshida, H. y otros tres autores; Support Effect on Methane Combustion Over Palladium Catalysts, Appl. Catal. B: Environmental: 71(1-2), 70-79 (2007). 
Zhimin, L. y otros cinco autores; Effect of $\mathrm{Fe}_{2} \mathrm{O}_{3}$ Loading Amount on Catalytic Properties of Monolithic $\mathrm{Fe}_{2} \mathrm{O}_{3} / \mathrm{Ce}_{0.67} \mathrm{Zr}_{0.33} \mathrm{O}_{2}-\mathrm{Al}_{2} \mathrm{O}_{3}$ Catalyst for Methane Combustion, J. of Rare Earths: 25(5), 585-589 (2007).

Zwinkels, M.F., S.G. Järas y P.Menon; Catalytic Materials for High Temperature Combustion, Catal. Rev. Sci. Eng.: 35(3), 319-358 (1993). 